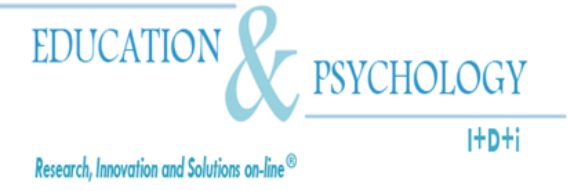

\title{
Efectos del análisis de textos sobre la elaboración y justificación de preguntas de investigación ${ }^{1}$
}

\author{
María Antonia Padilla Vargas ${ }^{2}$, Wendy Guadalupe Solór- \\ zano Sandoval ${ }^{3}$, Virginia Pacheco Chávez ${ }^{4}$ \\ ${ }^{2}$ Centro de Estudios e Investigaciones en Comportamiento, \\ Universidad de Guadalajara \\ ${ }^{3}$ Centro de Estudios e Investigaciones en Comportamiento, Universidad de \\ Guadalajara \\ ${ }^{4}$ Facultad de Estudios Superiores Iztacala, Universidad Nacional Autónoma de \\ México \\ Guadalajara, Jalisco
}

\section{México}

\footnotetext{
${ }^{1}$ Estudio llevado a cabo gracias al financiamiento de CONACYT, con el proyecto $46262-\mathrm{H}$.
}

$M^{a}$ Antonia Padilla Vargas. Centro de Estudios e Investigaciones en Comportamiento de la Universidad de Guadalajara. Francisco de Quevedo 180, Col. Arcos Vallarta. Guadalajara, Jalisco, México. C. P. 41130. E-mail: tony.padilla2008@gmail.com

C Education \& Psychology $\mathrm{I}+\mathrm{D}+\mathrm{i}$ and Editorial EOS (Spain) 


\section{Resumen}

Introducción. Se ha encontrado correspondencia entre el nivel en que se ejercita la lectura de textos científicos y el desempeño al escribir dicho tipo de textos. Además de leer en los niveles más complejos, formular un problema de investigación requiere instrucción explícita en escritura. El objetivo del presente estudio fue evaluar si identificar y elaborar diferentes tipos de párrafos de un artículo experimental, propiciaba que estudiantes de posgrado pudieran escribir una pregunta de investigación fundamentada.

Método. Participaron 11 alumnos de un posgrado en psicología. El grupo experimental se expuso a un entrenamiento consistente en identificar y completar, reformulando o parafraseando), algunos elementos de artículos experimentales. El grupo control no recibió ningún tipo de entrenamiento. La evaluación consistió en la elaboración y fundamentación de una pregunta de investigación.

Resultados. Los datos mostraron que antes del entrenamiento los estudiantes tuvieron severas dificultades en la elaboración y fundamentación de preguntas de investigación ya que ejercitaron los comportamientos lector y escritor en el nivel menos complejo, pero luego de exponerse al entrenamiento su desempeño mejoró sustancialmente, al lograr comportarse en niveles extrasituacionales. Los participantes del grupo experimental tuvieron un mejor desempeño que los del grupo control, tanto en la elaboración como en la fundamentación de sus preguntas de investigación.

Conclusión. Los resultados obtenidos en el presente estudio parecen indicar que exponer a investigadores en formación a la lectura de materiales complejos, y entrenarlos de manera explícita a escribir textos científicos promueve que éstos mejoren su desempeño al elaborar y justificar preguntas de investigación novedosas. Tales datos apuntan a la necesidad de proveer entrenamiento lector y escritor en niveles complejos a los científicos en formación.

Palabras clave: comportamiento lector, comportamiento escritor, elaboración y fundamentación, preguntas de investigación, estudiantes de posgrado. 


\begin{abstract}
Introduction. A correspondence between the level at which one can read scientific texts and his/her performance on writing this type of texts has been found. Besides reading at the most complex levels, raising research problems requires explicit training in writing. The objective of the present study was to evaluate whether identifying and elaborating different types of paragraphs of an experimental article improved graduate students' performance when asked to write a pertinent research question.
\end{abstract}

Method. Eleven psychology graduate students participated. The experimental group was exposed to a training consisting of identifying and rephrase some elements of an experimental article. The control group did not receive any type of training. The evaluation consisted of the elaboration and justification of a research question.

Results. Before training, the students showed severe difficulties in the elaboration and justification of research questions since they had exercised reading and writing only at less complex levels; after training, their performance improved substantially as they behaved at extrasituational levels. The participants of the experimental group performed better than those of the group control, both in the elaboration and in the justification of their research questions.

Conclusions. The results obtained in the present study seem to indicate that exposing researchers in formation to the reading of complex materials, and training them in an explicit way to write scientific texts improve their performance when elaborating and justifying novel research questions. Such data suggest the necessity to provide reading and writing training at more complex levels to novice researchers.

Key words: reading behavior, writing behavior, elaboration and justification, research questions, graduate students.

Received: 11/06/08_Initial Acceptance: 07/07/08_Definitive Acceptance: 07/01/09 


\section{Introducción}

Uno de los principales objetivos de la enseñanza de la ciencia es que los nuevos investigadores se desempeñen de manera efectiva y novedosa (De La Fuente, Justicia, Casanova y Trianes, 2005; Sánchez, 2004). Ello incluye que ejerciten habilidades escritoras, pues escribir textos especializados es parte de practicar una disciplina específica (Cassany, 2006). Según Keys, Hand, Prain y Collins (1999), la escritura de textos científicos, además de permitir la difusión del conocimiento y la evidencia empírica, promueve la generación de nuevo conocimiento.

Por otra parte, la lectura de textos científicos es una de las actividades indispensables al realizar investigación, ya que la práctica científica, debido a su naturaleza colectiva, requiere de constantes revisiones bibliográficas que permitan conocer lo que otros han hecho en el área de interés. De hecho, por lo general, es a partir de la revisión de dichos materiales que los investigadores plantean sus propias preguntas de investigación, derivadas de lo que ya se ha hecho en el área (Viniegra, 2002).

La investigación sobre las diversas variables involucradas en la lectura ha sido vasta y se ha hecho desde diferentes aproximaciones teóricas. Sin embargo, a pesar de su importancia en el ejercicio de la práctica científica, son pocos los estudios que analizan qué variables influyen en la conducta de escribir y más escasos aún los trabajos que estudian la relación entre las habilidades lectoras desarrolladas por los científicos y sus aprendices, y las habilidades escritoras desplegadas en una tarea específica. De ahí la relevancia de hacer investigaciones en esta dirección (Carpio e Irigoyen, 2005; Pacheco, Ramírez, Palestina y Salazar, 2007).

El presente trabajo se ubica en el marco de la psicología interconductual, con base en el cual la lectura comprensiva se define como una función psicológica en la que existe una relación entre el sujeto que comprende, lo que es comprendido y las condiciones mínimas necesarias para que la relación entre estos dos elementos se establezca (Fuentes, 2005). Se dice que alguien comprendió cuando su comportamiento guarda correspondencia con las diferentes situaciones caracterizadas como comprensión. Carpio, Pacheco, Flores y Canales (2000) plantean que el ajuste lector refiere la adecuación del comportamiento a las demandas de una situación específica, plasmadas en un texto. Tales demandas o criterios de ajuste pueden involucrar requerimientos conductuales de complejidades diferentes (Carpio, 1994; Ribes, 2004). 
Leer y escribir se pueden ejercitar en diferentes niveles de desligamiento funcional progresivamente más complejos e inclusivos (Ribes y López, 1985). Los niveles son:

1) Nivel situacional diferencial: En este nivel el individuo responde a las regulaciones espacio-temporales de los objetos de estímulo sin alterarlas. Un ejemplo de este nivel de interacción tiene lugar cuando un niño dice "Coca Cola" cuando ve un envase de refresco que tiene tales letras impresas, o cuando copia ese texto.

2) Nivel situacional efectivo: En este nivel el individuo, con su actividad, modifica el ambiente eliminando objetos o eventos en la situación presente. Un ejemplo en este nivel es cuando un estudiante lee reportes de investigación e identifica y transcribe elementos como definiciones, evidencias, etc.

3) Nivel situacional preciso: Este nivel implica que el sujeto diferencie las relaciones contextuales cambiantes, con base en la dimensión de significación funcional vigente de los eventos y organismos del entorno. Un ejemplo de comportamiento en este nivel es cuando un estudiante clasifica, con base en una tipificación o taxonomía que se le entregue para tal fin, los párrafos que componen la introducción o la discusión de un artículo leído previamente.

4) Nivel extrasituacional: En este nivel el individuo altera las relaciones entre objetos e individuos de la situación concreta presente con base en las contingencias de otra situación concreta (pasada, futura o distante). Un ejemplo de comportamiento de este tipo ocurre cuando un alumno reformula (parafraseando) la introducción o la discusión de un artículo de investigación, o cuando escribe un proyecto propio derivado de uno o más artículos científicos leídos previamente.

5) Nivel transituacional: En este nivel el individuo interactúa en términos de conducta convencional y sus productos, lo cual implica hacer contacto con sistemas convencionales abstractos, aplicables a diversas situaciones y no a una en particular. Un ejemplo de una conducta en este nivel es cuando un estudiante propone un nuevo modelo o taxonomía para explicar un fenómeno a partir de lo leído en una cierta área de conocimiento (Pacheco, Ramírez, Palestina y Salazar, 2007).

Se han llevado a cabo algunos estudios en los que se ha analizado el comportamiento lector. La tarea experimental básica empleada implica exponer a estudiantes universitarios a diferentes tareas con el fin de evaluar su comprensión lectora en los cinco niveles funcionales previamente mencionados. Los resultados indican que el mayor número de aciertos se obtiene en los niveles menos complejos, mientras que el peor desempeño se presenta en las tareas que demandan a los participantes leer en niveles extra y transituacionales (Canales, Morales, 
Arroyo, Pichardo y Pacheco, 2005; Carpio e Irigoyen, 2005; Irigoyen, Jiménez y Acuña, 2004).

Uno de los principales objetivos de la práctica científica es generar conocimiento. Para ello es indispensable que los científicos sean capaces de leer y escribir en todos los niveles funcionales, especialmente en los mas complejos, ya que para generar conocimiento es necesario por lo menos comportarse de manera extrasituacional, lo cual implica identificar los distintos elementos que componen un texto, relacionarlos con lo previamente leído, así como argumentar y derivar preguntas de investigación novedosas, justificándolas o fundamentándolas a partir de los materiales técnicos revisados (Fuentes, 2005).

Si bien no son condición suficiente, las habilidades lectoras son indispensables para el desarrollo de la conducta de escribir (Pacheco, 2008). Al respecto, Cassany (2007) sostiene que "escribir exige un refinado y diverso dominio de las destrezas de la lectura" (p. 39). Ribes, Cortés y Romero (1992), al hablar acerca de la evolución psicológica de las interacciones lingüísticas (leer y escribir entre ellas) hacia los niveles más complejos (extra y transituacionales), sostienen que el individuo, al participar en los sistemas reactivos convencionales, desarrolla morfologías y las ejercita en situaciones y dominios específicos. Afirman que la participación del individuo en los sistemas reactivos convencionales ocurre siempre como configuración de morfologías convencionales y no convencionales en circunstancias específicas (ante objetos y eventos específicos).

Según Ribes (1990), las condiciones de tal evolución psicológica son: 1) Una historia interactiva que involucre sistemas reactivos convencionales en los niveles de desligamiento intrasituacionales; 2) "La aptitud para ser mediado por las respuestas convencionales de otro individuo en relación con las contingencias de y entre los eventos fisicos y sociales no presentes en la situación, no directamente observables o aparentes... 3) El entrenamiento explícito del individuo para interactuar como mediador respecto de otros individuos, considerando las propiedades no presentes como contingencias actualmente disponibles" (Ribes, 1990, p. 169).

La conducta de leer, vinculada con la segunda condición evolutiva descrita arriba, implica que al leer en los niveles más complejos, un individuo hace contacto funcional con un referente, a través del texto elaborado por otro individuo. El individuo que lee hace contacto 
con el referente y con la manera como se refiere en dominios específicos (morfologías, estilos, sintaxis), lo cual puede favorecer que ese individuo medie por escrito otros referentes. En correspondencia con lo anterior, leer solo en niveles intrasituacionales hace menos probable que un individuo logre mediar diferentes tipos de contingencias por escrito (por ejemplo, formular preguntas de investigación). De lo anterior puede derivarse que el desempeño del individuo como lector está relacionado con su desempeño efectivo como escritor (Pacheco et al., 2007).

Pacheco et al. (2007), realizaron una investigación con el propósito de analizar una posible relación entre el comportamiento lector y escritor. Se pidió a estudiantes de una licenciatura en psicología que leyeran un artículo científico y que llevaran a cabo una serie de actividades: elaborar un resumen, emitir una opinión acerca del texto leído, identificar elementos de dicho texto, plantear una pregunta de investigación derivada de éste, y proponer un anteproyecto de investigación. Encontraron que la mayoría de los participantes se desempeñaron mejor en las tareas de lectura y escritura que implicaban niveles de interacción situacionales, que cuando la actividad requería que se comportaran en un nivel más complejo. Lo anterior parece indicar que interactuar con el texto en un nivel extrasituacional es necesario para ejercitar la escritura en dicho nivel.

Aparentemente, leer en un nivel extrasituacional permite al estudiante establecer relaciones entre diversos elementos contenidos en el texto leído, lo cual posibilita plantear preguntas de investigación novedosas (Pacheco et al., 2007). Una pregunta de investigación se considera novedosa en la medida en que en ésta se cambie como mínimo el valor de una de las variables que se propone evaluar. Dicho cambio sería con respecto al(os) reporte(s) de investigación o texto(s) del(os) que dicha pregunta se haya derivado.

Como se mencionó previamente, de acuerdo con Ribes et al. (1992), además de una historia interactiva en los niveles intrasituacionales, y aprender a ser mediado por las respuestas convencionales de otros, para interactuar como mediador respecto de otros, el individuo requiere necesariamente ser expuesto a un entrenamiento en el que se le enseñe de manera explícita a identificar las propiedades no presentes o no aparentes de una situación.

Sin embargo, en el caso de la conducta de escribir, la instrucción explícita no es lo común. Pacheco (2008), reportó que en el caso de la formación de psicólogos a nivel de pre- 
grado en la FES Iztacala de la UNAM, la enseñanza y el aprendizaje de habilidades escritoras suele ser mas un efecto colateral, y en consecuencia poco sistematizado, que un objetivo concreto de los programas curriculares. Por ello, resalta la necesidad de diseñar e implementar de manera explícita herramientas didácticas dirigidas a que los estudiantes desarrollen habilidades específicas para elaborar proyectos de psicología experimental, asunto éste planteado reiteradamente en la literatura del área (Cassany, 2006).

Considerando que lo anterior es aplicable a los cursos de posgrado, la presente investigación se llevó a cabo con el objeto de analizar los efectos de entrenar a clasificar y a reformular diferentes tipos de párrafos, sobre el comportamiento escritor, específicamente sobre la elaboración y fundamentación de preguntas de investigación psicológica, en estudiante de posgrado.

Bajo el supuesto que en la evolución de las interacciones extrasituacionales los individuos aprenden inicialmente a ser mediados, es decir, a hacer contacto con los referentes y con la manera de referirlos, y que progresivamente, con base en la experiencia que van adquieriendo, pueden aprender a ser mediadores de referentes eventualmente diferentes, se diseñó un entrenamiento que requería que los participantes aprendieran primero a identificar algunos elementos que conforman la sección introductoria de un reporte de investigación, tales como definiciones de conceptos básicos, evidencias experimentales, hipótesis, y diferentes preguntas de investigación y objetivos experimentales. Y posteriormente, que aprendieran a reformular tales elementos componentes, con el objetivo final de auspiciar que derivaran preguntas de investigación novedosas, pertinentes y debidamente fundamentadas, a partir de los textos previamente leídos.

La hipótesis del presente estudio es que el entrenamiento en lectura comprensiva y en composición escrita de textos científicos, en el nivel extrasituacional, promovería la elaboración y fundamentación de preguntas de investigación en dicho nivel, en estudiantes de primer semestre de posgrado. 


\section{Método}

\section{Participantes}

La muestra seleccionada para el presente estudio estuvo compuesta por todos los alumnos que iniciaron en el ciclo escolar 2007 de un posgrado en ciencias del comportamiento. Dicho posgrado tenía como objetivo la formación de nuevos investigadores en el área de psicología experimental. Tenía un plan curricular que funcionaba con base en un sistema tutorial. Con el objeto de poder entrenarlos de manera personalizada, cada semestre se permitía sólo el ingreso de un número reducido de alumnos. En el semestre elegido ingresaron un total de 11; 5 hombres y 6 mujeres, de entre 23 y 30 años. Los participantes se distribuyeron al azar en dos grupos, uno experimental, compuesto por 6 alumnos ( 3 hombres y 3 mujeres), y uno control, compuesto por 5 (2 hombres y 3 mujeres). Ninguno de los participantes había sido expuesto a tareas similares a las empleadas en el presente estudio. Es decir, no habían sido entrenados para identificar elementos de textos científicos, ni para reelaborar apartados de éstos, así como para elaborar y fundamentar preguntas de investigación. Debido a que el objetivo del presente estudio era exponer a la preparación experimental diseñada a todos los alumnos que ingresaran en un ciclo escolar determinado, y a que el número de éstos era reducido, no se controló a priori, aunque sí se identificó, la experiencia que los participantes tenían en la realización de actividades de investigación. Para identificar su nivel de entrada en comprensión lectora y composición escrita se aplicó una Línea Base (LB). La participación fue voluntaria.

\section{Instrumentos}

Para la LB, el entrenamiento y la evaluación de la comprensión lectora y de la composición escrita se utilizaron textos de artículos científicos (del área interconductista) y materiales preparados ex profeso (éstos se describen en el siguiente apartado). La temática de dichos artículos giraba en torno a la implementación de preparaciones experimentales que permitieran identificar las variables involucradas en la resolución de problemas complejos, como por ejemplo, analizaban la generación de conducta creativa, los efectos de exponerse a diferentes tipos de retroalimentación, o las condiciones que permitieran el establecimiento de relaciones de quivalencia. En el Anexo 1 se muestra la lista de los artículos que fueron empleados. 


\section{Diseño}

Se formaron dos grupos al azar: uno experimental y uno control. El grupo experimental fue expuesto a una LB (pretest), a los ejercicios de entrenamiento 1 y 2 (intervención), y a una evaluación con retroalimentación (postest), mientras que el grupo control se expuso a la LB, a la lectura de los artículos empleados en los ejercicios 1 y 2 con el grupo experimental, y a una evaluación sin retroalimentación. A los participantes del grupo control se les pedía que leyeran los artículos empleados en los ejercicios 1 y 2 debido a que en la evaluación debían derivar su pregunta de investigación de tales artículos (dado que como medida de control era necesario que ambos grupos derivaban sus preguntas de los mismos artículos), además de que se quería probar que la sola lectura de tales materiales no propiciaba que los sujetos fueran capaces de elaborar y fundamentar preguntas de investigación. Las sesiones experimentales se realizaron en cubículos sonoamortiguados de dos metros cuadrados, que contaban con iluminación natural y eléctrica. En cada cubículo había una mesa, una silla y un equipo de cómputo. En la Tabla 1 se presenta el diseño experimental.

Tabla 1.Diseño experimental empleado.

\begin{tabular}{|c|c|c|c|c|}
\hline & CONDICIO & & & \\
\hline & Pretest & Entrenamiento (Inte & vención) & Postest \\
\hline & & Ejercicio 1 & Ejercicio 2 & \\
\hline Grupo Expe- & Línea Base & 1) Leer artículos & 1) Leer artículos & Evaluación \\
\hline rimental & & 2) Realizar ejerci- & 2) Realizar ejerci- & \\
\hline$N=6$ & & $\begin{array}{l}\text { cios de identifica- } \\
\text { ción }\end{array}$ & $\begin{array}{l}\text { cios de elabora- } \\
\text { ción }\end{array}$ & \\
\hline Grupo Control & Línea Base & 1) Leer artículos & 1) Leer artículos & Evaluación \\
\hline $\mathrm{N}=5$ & & & & \\
\hline
\end{tabular}

\section{Procedimiento}

Los participantes se expusieron a diferentes condiciones: a una LB, a 2 bloques de ejercicios de entrenamiento y a una evaluación. En la LB los participantes debían identificar los distintos elementos que componen un artículo científico y elaborar y fundamentar una 
pregunta de investigación derivada de los artículos previamente leídos. En el Ejercicio 1 debían identificar y clasificar (nivel situacional) los tipos de párrafos que componen un artículo. En el Ejercicio 2 debían completar, reformulando o parafraseando (nivel extrasituacional), algunos párrafos de la introducción de cada uno de los artículos previamente leídos, además de identificar el tipo de pregunta de investigación que éstos pretendían contestar, así como identificar las variables evaluadas en éste. Mientras que en la Evaluación debían elaborar y fundamentar (nivel extrasituacional) una pregunta de investigación novedosa y pertinente, a partir de los textos leídos en el Ejercicio 2.

Los grupos experimental y control se diferenciaban en que los participantes del grupo control no se exponían a los ejercicios de entrenamiento, aunque sí debían leer los artículos que los del grupo experimental leían para realizar tales ejercicios. Lo anterior se hizo de esa manera para que la única diferencia entre ambos grupos fuera la exposición a los ejercicios de entrenamiento. A continuación se detalla en qué consistió cada una de las condiciones a las que se expuso a los participantes.

Línea Base. Cada participante debía leer dos artículos científicos y con base en lo leído, debía escribir la introducción a un anteproyecto y elaborar un esquema de éste. Luego debía identificar, en uno de los textos leídos: los autores, el título, las variables independiente y dependiente, los conceptos centrales, las evidencias, los resultados y las conclusiones. Posteriormente debía elaborar y fundamentar una pregunta de investigación derivada de los artículos leídos. Vale aclarar que la LB y la evaluación diferían en que en la evaluación sólo debían elaborar y fundamentar una pregunta de investigación, mientras que en la LB, además de lo anterior, debían llevar a cabo las actividades previamente descritas. Ello se hizo así debido a que en un estudio previo se detectó que cuando no se llevaban a cabo dichas actividades adicionales en la LB, un gran número de participantes se declaraba incapaz de realizar la tarea y desertaba del estudio argumentando que no sabían como elaborar, y sobre todo fundamentar, una pregunta de investigación.

Ejercicios 1 y 2: El entrenamiento al que se expuso a los participantes del grupo experimental constó de los ejercicios 1 y 2 . Con el objeto de asegurarse de que el entrenamiento fuera suficiente para que el participante aprendiera lo requerido, cada ejercicio constaba de 3 sesiones similares (sólo diferían en que para cada una se empleaba un artículo diferente). En el Ejercicio 1 el participante debía: a) clasificar los párrafos que conforman la introducción de 
un artículo experimental de psicología (identificando si se trataba de definiciones, evidencias, planteamientos derivados, formulación de preguntas y objetivos, o una combinación de los anteriores); y b) leer la definición de una variable independiente y una dependiente. Mientras que en el Ejercicio 2 el participante debía: a) leer un artículo, b) identificar que los párrafos que conforman la introducción de un artículo experimental de psicología se vinculan a partir de la pregunta de investigación; c) identificar el tipo de pregunta de investigación que se planteaba en cada uno de los artículos que leía (con base en criterios que se describen en el siguiente apartado); y d) completar (reformulando o parafraseando) algunos párrafos del artículo leído, y finalmente, e) identificar las variables independiente y dependiente planteadas en éste. Al final de cada una de las sesiones de ambos ejercicios se daba retroalimentación a los participantes respecto de su desempeño.

Para la parte del inciso $b$, consistente en la identificación del tipo de pregunta de investigación que se planteaba en cada uno de los artículos que debían leer, se les proporcionó la tipificación de la Tabla 3, en la que se enlistan los tipos de preguntas que es posible elaborar. Según dicha clasificación, los niveles en que se puede plantear una pregunta son inclusivos y van de menor a mayor complejidad, lo que implica que el investigador despliegue un comportamiento cada vez mas desligado de las propiedades concretas de la situación que se haya planteado en el reporte(s) experimental(es) que haya revisado para derivar a partir de ahí su propia pregunta. Lo que los participantes del grupo control hacían en esta parte era leer los mismos artículos revisados por los del grupo experimental, así como leer la definición de una variable independiente y una dependiente. Los ejercicios 1 y 2 se describen en la Tabla 3.

Tabla 2. Descripción de los Ejercicios 1 y 2 que formaban parte del entrenamiento.

\begin{tabular}{|c|c|c|c|}
\hline & & Grupo Experimental & Grupo Control \\
\hline $\begin{array}{l}\overrightarrow{0} \\
\frac{0}{0} \\
\frac{0}{0} \\
\frac{0}{0} \\
\frac{0}{\omega 0}\end{array}$ & $\begin{array}{l}\text { Sesión } 1 \\
\text { Sesión } 2\end{array}$ & $\begin{array}{l}\text { a) Leer un artículo previamente clasifica- } \\
\text { do, b) clasificar los párrafos que confor- } \\
\text { man la introducción de un artículo, y c) } \\
\text { leer la definición de una variable inde- } \\
\text { pendiente y una dependiente. } \\
\text { Similar a la sesión anterior, pero con un } \\
\text { artículo diferente. }\end{array}$ & $\begin{array}{l}\text { a) Leer un artículo (el mismo } \\
\text { leído por los participantes del } \\
\text { grupo experimental), y b) leer la } \\
\text { definición de una variable inde- } \\
\text { pendiente y una dependiente, } \\
\text { Similar a la sesión anterior, pe- } \\
\text { ro con un artículo diferente. }\end{array}$ \\
\hline
\end{tabular}




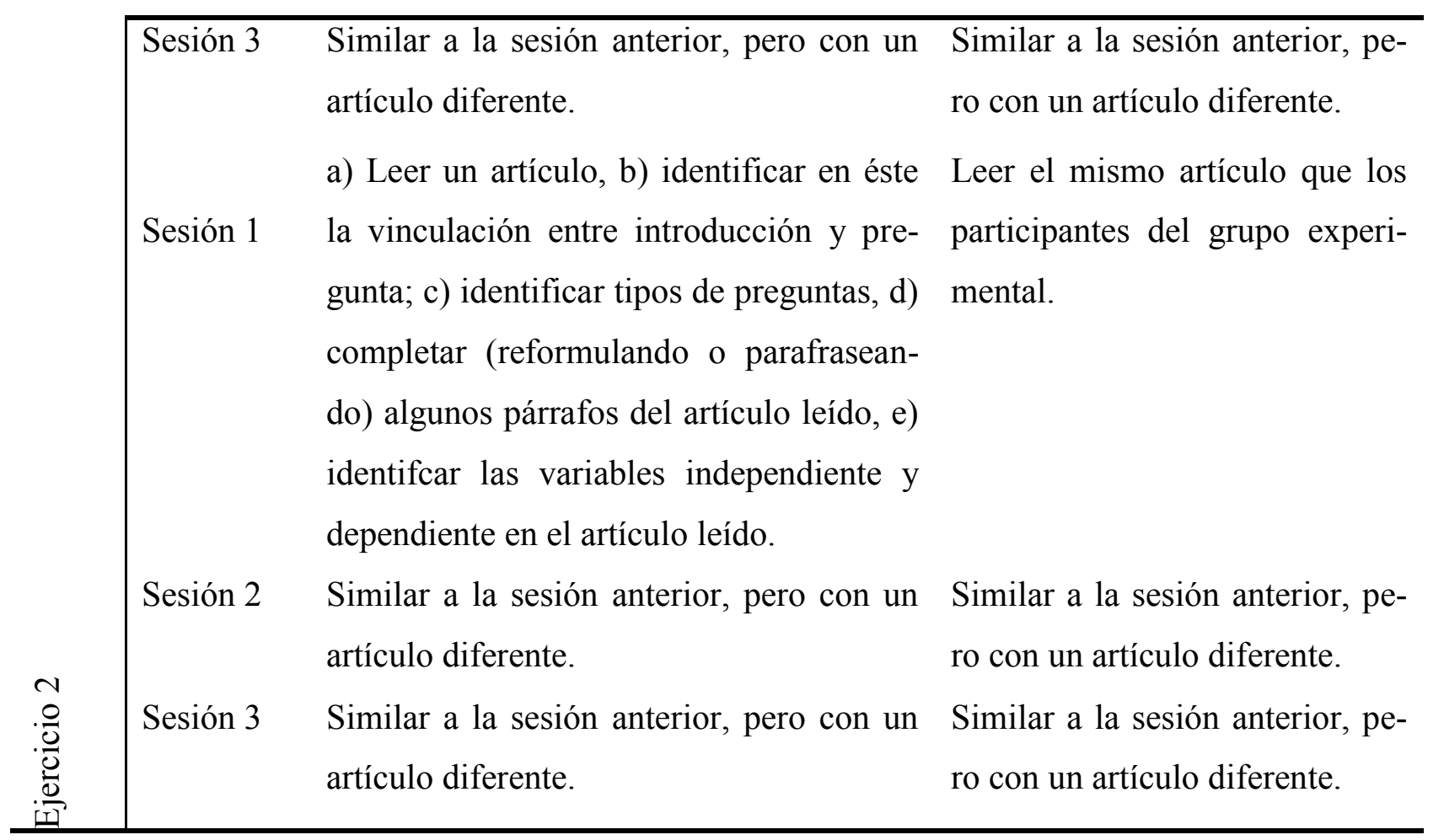

Tabla 3. Tipificación de preguntas de investigación (Pacheco, 2007).

1. Se manipulan variables ya evaluadas y sólo se cambia el valor de una de las variables.

2. a) Se evalúan relaciones (entre variables) no consideradas en la literatura del área de conocimiento, b) se manipulan variables no evaluadas y que son pertinentes al problema de interés, y c) se aplican los principios de una teoría científica para explicar un problema social concreto (relacionado con la investigación tecnológica) o un problema científico (relacionado con la investigación básica).

3. Se proponen preparaciones experimentales que permiten validar los hechos de una teoría.

Evaluación. Esta condición constaba de dos sesiones que se describen en la Tabla 4. En la primera sesión los participantes debían elaborar y fundamentar una pregunta de investigación novedosa y pertinente, derivada de alguno o todos los textos leídos en el Ejercicio 2. En cuanto concluían dicha tarea se les retroalimentaba su desempeño (sólo a los participantes del grupo experimental), luego de lo cual pasaban a la sesión 2, que consistía en la corrección de los errores que se les hubieran señalado. Se decidió proporcionar retroalimentación y hacer correctiva esta parte sólo para los participantes del grupo experimental debido a que se quería 
probar el efecto de exponer a los sujetos a entrenamiento en lectura comprensiva y en composición escrita (grupo experimental), versus el sólo hecho de leer artículos o releer lo escrito por el propio sujeto, sin que éste recibiera ningún tipo de entrenamiento, ni de retroalimentación, ni que debiera corregir sus errores (situación a la que se expuso a los participantes del grupo control).

Los datos que se analizaron fueron: de la LB, la identificación de los distintos elementos que componen un artículo, así como la pregunta de investigación que elaboraban y la fundamentación de ésta. Del Ejercicio 1, la identificación y clasificación del tipo de párrafos que componían la introducción de los artículos revisados; del Ejercicio 2, la identificación de los tipos de preguntas planteadas, la elaboración de las paráfrasis y la identificación de las variables dependiente e independiente, en cada uno de los artículos revisados; y de la Evaluación, la elaboración y fundamentación de la pregunta de la investigación, antes y después de la retroalimentación. Se obtuvieron los siguientes datos: a) de la LB, el número de elemenos identificados correctamente del artículo que se les entregó para tal fin; de la LB y de la Evaluación, el número de aciertos obtenido por cada sujeto en la fundamentación de la pregunta de investigación, si elaboraban o no una pregunta de investigación y en qué nivel era ésta planteada; y b) de los ejercicios 1 y 2 , el número de aciertos obtenido por cada sujeto en cada una de las 3 sesiones que los componían.

Tabla 4. Esquema del procedimiento empleado en la Evaluación.

\begin{tabular}{|c|c|c|c|}
\hline & Grupo Experimental & Grupo Control \\
\hline 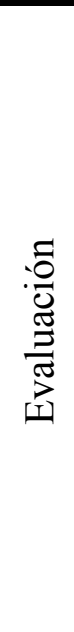 & Sesión 1 & $\begin{array}{l}\text { Leer los artículos completos del Ejerci- } \\
\text { cio } 2 \text {, derivar una pregunta de investiga- } \\
\text { ción, redactar la introducción de su an- } \\
\text { teproyecto, clasificar párrafos y pregunta } \\
\text { planteada, y definir VD y VI. } \\
\text { Reelaborar pregunta de investigación y } \\
\text { su correspondiente fundamentación a } \\
\text { partir de la retroalimentación recibida. }\end{array}$ & $\begin{array}{l}\text { Leer los artículos completos del } \\
\text { Ejercicio 2, derivar una pregunta } \\
\text { de investigación, y redactar la } \\
\text { introducción de su anteproyecto. } \\
\text { Reelaborar pregunta de investi- } \\
\text { gación y su correspondiente fun- } \\
\text { damentación sin recibir retroali- } \\
\text { mentación. }\end{array}$ \\
\hline
\end{tabular}


Los datos obtenidos en la LB y en la Evaluación se analizaron de la siguiente forma: la fundamentación de la pregunta de investigación se evaluó en términos de si ésta cumplía o no con los párrafos correspondientes a los siguientes elementos: título, definiciones, evidencias, planteamientos derivados y formulación de pregunta y objetivo. Se asignaba un punto si la respuesta era suficiente, cero punto cinco si era insuficiente, y cero si era inadecuada o estaba ausente. Además, se calificaba con un punto si el participante lograba identificar correctamente la variable dependiente y otro punto si lograba identificar la variable independiente en la pregunta y fundamentación que elaboraba. Se sumaba el puntaje total obtenido y se obtenía el porcentaje de aciertos. Con base en la tipificación de Pacheco (comunicación personal, 2007) se identificó en qué nivel había sido planteada la pregunta de investigación elaborada por cada participante (ver Tabla 3).

Con respecto a los datos recabados en cada una de las sesiones de los ejercicios 1 y 2 , en las que los participantes debían identificar y clasificar párrafos en el caso del Ejercicio 1, e identificar el tipo de pregunta, reformular algunas partes del artículo revisado e identificar las variables indepediente y dependiente de éste, en el caso del Ejercicio 2, se otorgaba un punto por cada respuesta correcta, medio punto si la respuesta era correcta pero insuficiente y cero puntos si era inadecuada o estaba ausente. Se sumaba el puntaje total obtenido y se obtenía el porcentaje de aciertos. El análisis de todos los datos recabados fue sometido a una prueba de confiabilidad a través de dos calificadores independientes, dando una confiabilidad promedio de $98.80 \%(\%$ Confianza $=1-[(\mathrm{n} 1-\mathrm{n} 2) /(\mathrm{n} 1+\mathrm{n} 2)] * 100)$.

\section{Resultados}

Para considerar como válidas las preguntas de investigación elaboradas éstas tenían que ser experimentales y plantear una interacción entre una variable independiente y una dependiente. Un participante podía elaborar una pregunta que no calificara como tal, pero en su fundamentación, dado que se evaluaban varios aspectos (que se detallan en el apartado de procedimiento), podía lograr cierto puntaje de aciertos.

En lo relativo a los puntajes logrados por los participantes al identificar los distintos elementos que componen un artículo científico, en promedio los del grupo experimental obtuvieron $68 \%$ de aciertos, mientras que los del grupo control lograron $86 \%$. 
En lo que respecta a la elaboración de su pregunta de investigación, como puede observarse en la Figura 1, en la LB sólo dos participantes lograron plantearla, y ambos lo hicieron en el nivel 1. Con respecto a la fundamentación de la pregunta de investigación elaborada, como la Figura 2 muestra, los puntajes de ambos grupos fluctuaron entre $20 \%$ y $40 \%$ de aciertos, excepto por uno de los participantes del grupo experimental que obtuvo 60\% (P5) y uno del control que obtuvo 10\% (P11).
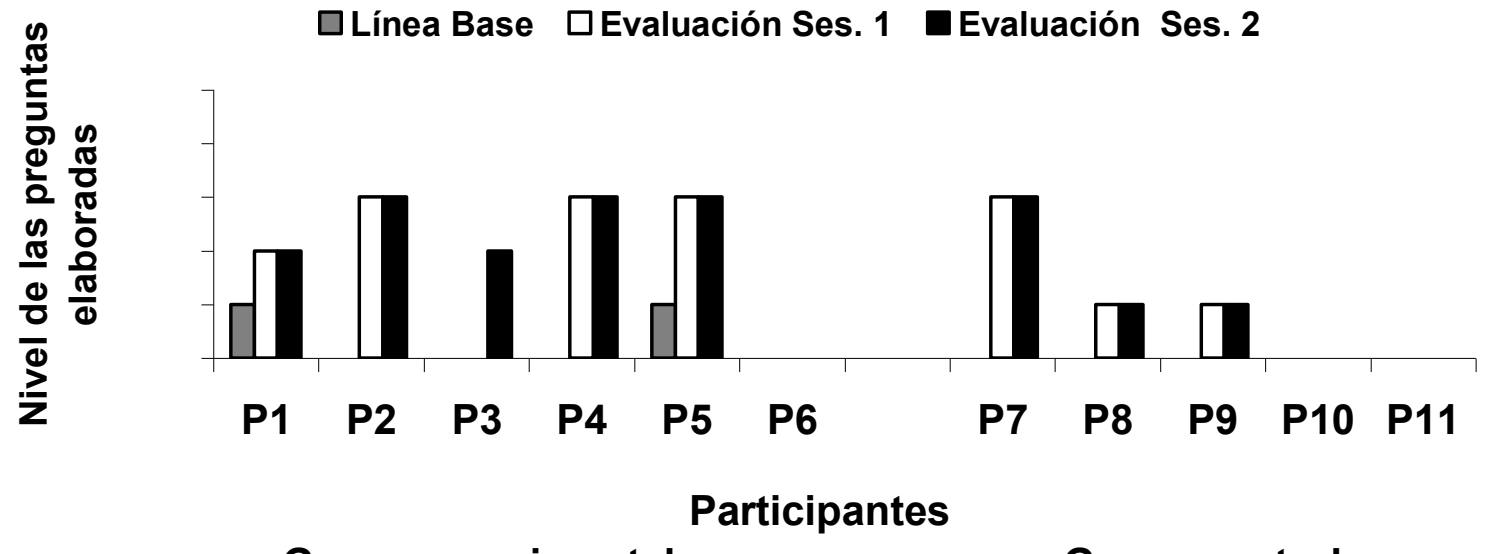

Grupo experimental

Grupo control

Figura 1. Niveles en que fueron planteadas las preguntas de investigación, por cada participante, en la Línea Base y en las Sesiones 1 y 2 de la Evaluación.

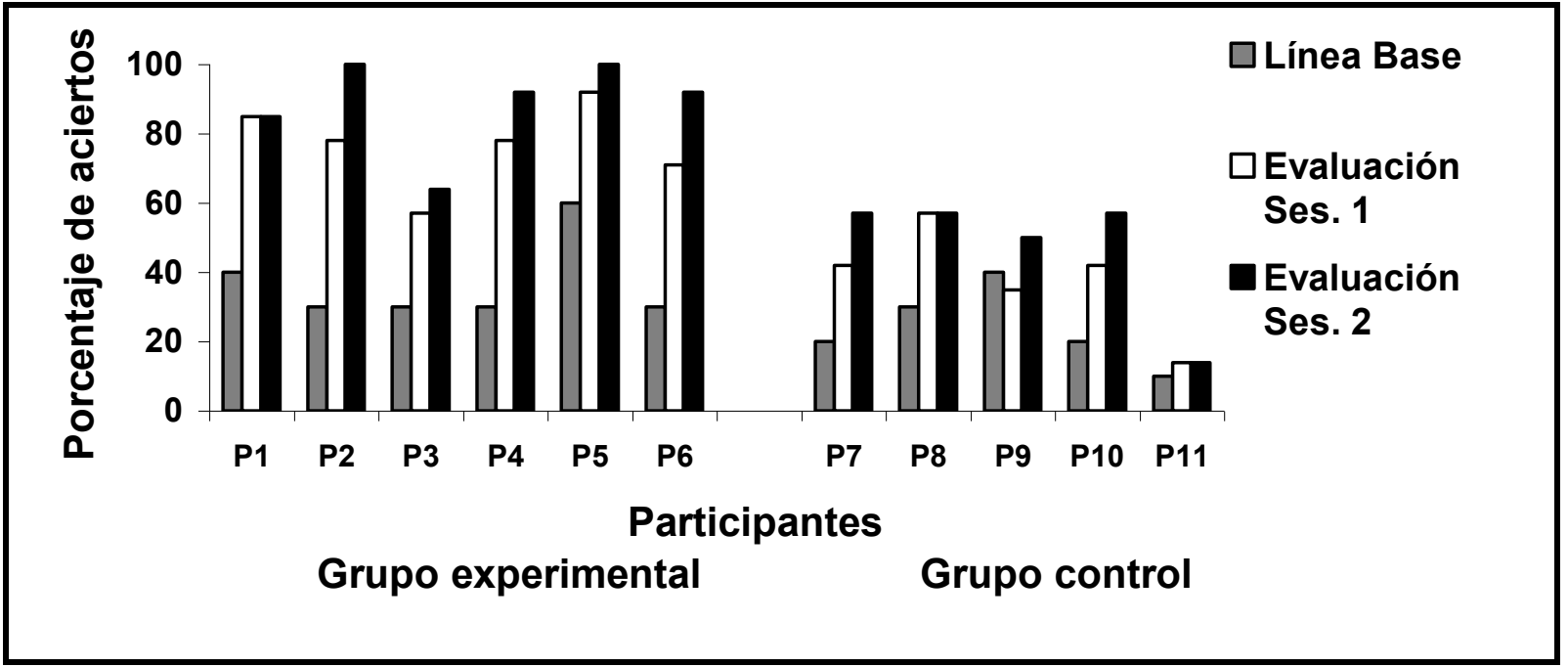

Figura 2. Porcentaje de aciertos obtenido en la fundamentación de la pregunta de investigación correspondiente a la Línea Base y a las Sesiones 1 y 2 de la Evaluación.

En la Figura 3 se graficó el porcentaje de aciertos logrado por cada participante en cada una de las sesiones del Ejercicio 1. Todos los participantes obtuvieron el mayor porcentaje 
de aciertos en la sesión 1, a excepción de los sujetos 3 y 4, quienes lograron el mayor porcentaje de aciertos en la sesión 3. Y todos obtuvieron los puntajes más bajos en la sesión 2.

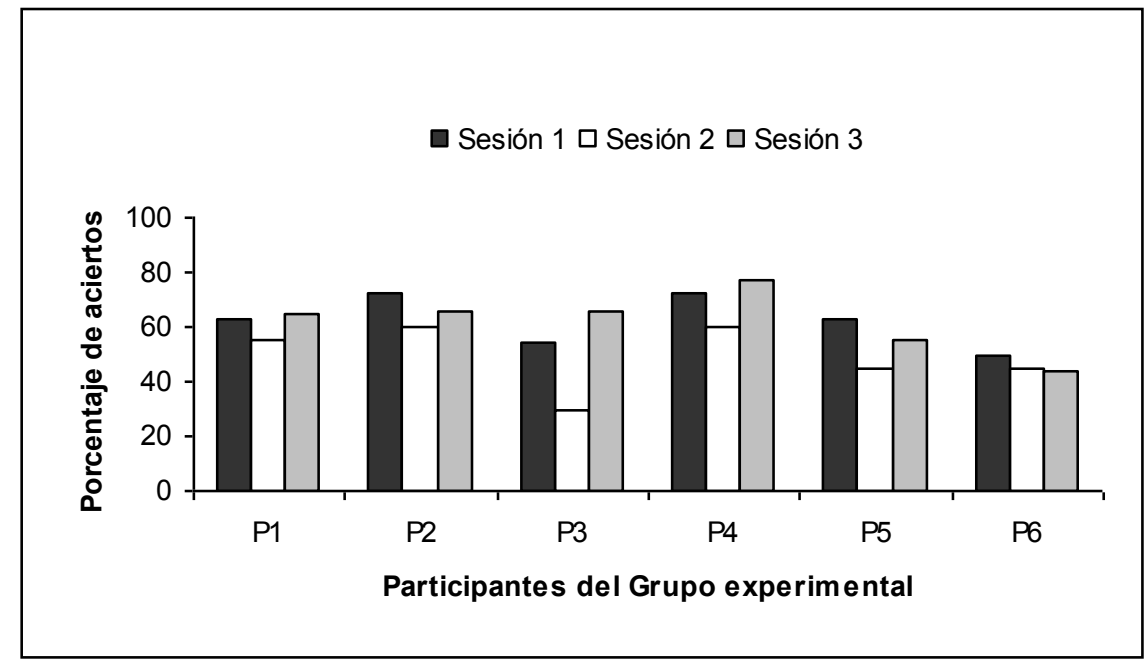

Figura 3. Porcentaje de aciertos obtenido por cada participante del Grupo Experimental en cada una de las sesiones del Ejercicio 1.

El porcentaje de aciertos obtenido en cada una de las sesiones del Ejercicio 2 se muestra en la Figura 4. Puede observarse que a excepción del participante 2, todos lograron un mayor puntaje de aciertos en la sesión 1. Y todos mostraron su menor porcentaje en la sesión 2, logrando incrementar su desempeño en la sesión 3.

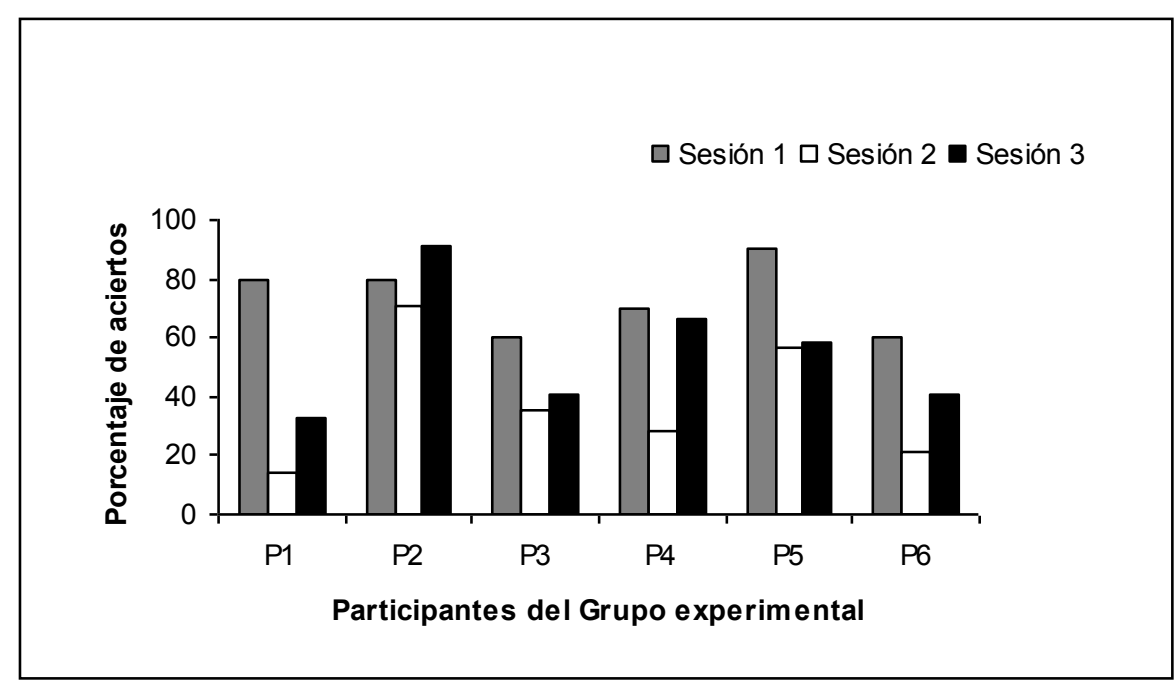

Figura 4. Porcentaje de aciertos obtenido por cada participante del Grupo Experimental en cada una de las sesiones del Ejercicio 2. 
En la Figura 1 se muestra el nivel en el que cada participante planteó sus preguntas de investigación en la LB y en cada una de las dos sesiones de la Evaluación. Se observa que a excepción de los participantes 6 (del grupo experimental), 10 y 11 (del grupo control), quienes no lograron elaborar una pregunta de investigación, todos fueron capaces de formularla en la Evaluación. Y quienes en la LB lo habían logrado en el nivel 1, en la Evaluación lo hicieron en el nivel 2a o 2b. Como lo muestra dicha figura, luego del entrenamiento los sujetos del grupo experimental elaboraron sus preguntas en niveles superiores ( $2 \mathrm{a}$ y $2 \mathrm{~b}$ ) a los del grupo control, quienes, o no elaboraron preguntas o lo hicieron en el nivel 1 (con excepción del P7, que lo hizo en el nivel 2b), sin embargo, tales diferencias no fueron significativas (Fisher's exact test, $\mathrm{p}=.455)$.

En cuanto a la fundamentación de la pregunta de investigación, en la Figura 2 se observa que tanto el grupo control como el experimental mostraron un mayor porcentaje de aciertos en la Evaluación que en la LB. Sin embargo, se observó un mayor porcentaje de ganancia por parte del grupo experimental, ya que en la LB su desempeño estuvo por debajo del $60 \%$ de aciertos, y en la Evaluación éste se acercó al 100\%, en contraste con el desempeño de los participantes del grupo control quienes obtuvieron porcentajes por debajo del $40 \%$ de aciertos en la LB y en la Evaluación cercanos al 60\%. Con el objeto de verificar si las diferencias observadas entre los grupos experimental y control eran estadíticamente significativas, se corrió la prueba estadística ANOVA para medidas repetidas. Se observó un efecto de fase para ambos grupos $(\mathrm{F}(2,18)=58.86, \mathrm{p}<.05)$. Dicho efecto fue más marcado para el grupo experimental, lo cual se observa en la interacción entre fase y grupo $(\mathrm{F}(2,18)=10.07, \mathrm{p}=$ .001). Además, se observaron diferencias estadísticamente significativas entre los grupos ( $\mathrm{F}$ $(1,9)=17.81, \mathrm{p}=.002)$.

Con el objeto de facilitar la comparación de los datos, se promedió el porcentaje de aciertos que los participantes de los grupos experimental y control obtuvieron al fundamentar la pregunta de investigación que elaboraron en la LB y en las dos sesiones de la Evaluación. Como puede verse en la Figura 5, en la LB los puntajes obtenidos por ambos grupos fueron similares (37\% y 24\%, respectivamente), mientras que en la Evaluación, tanto en la sesión 1 como en la 2, el grupo experimental obtuvo prácticamente el doble de aciertos que el control. 


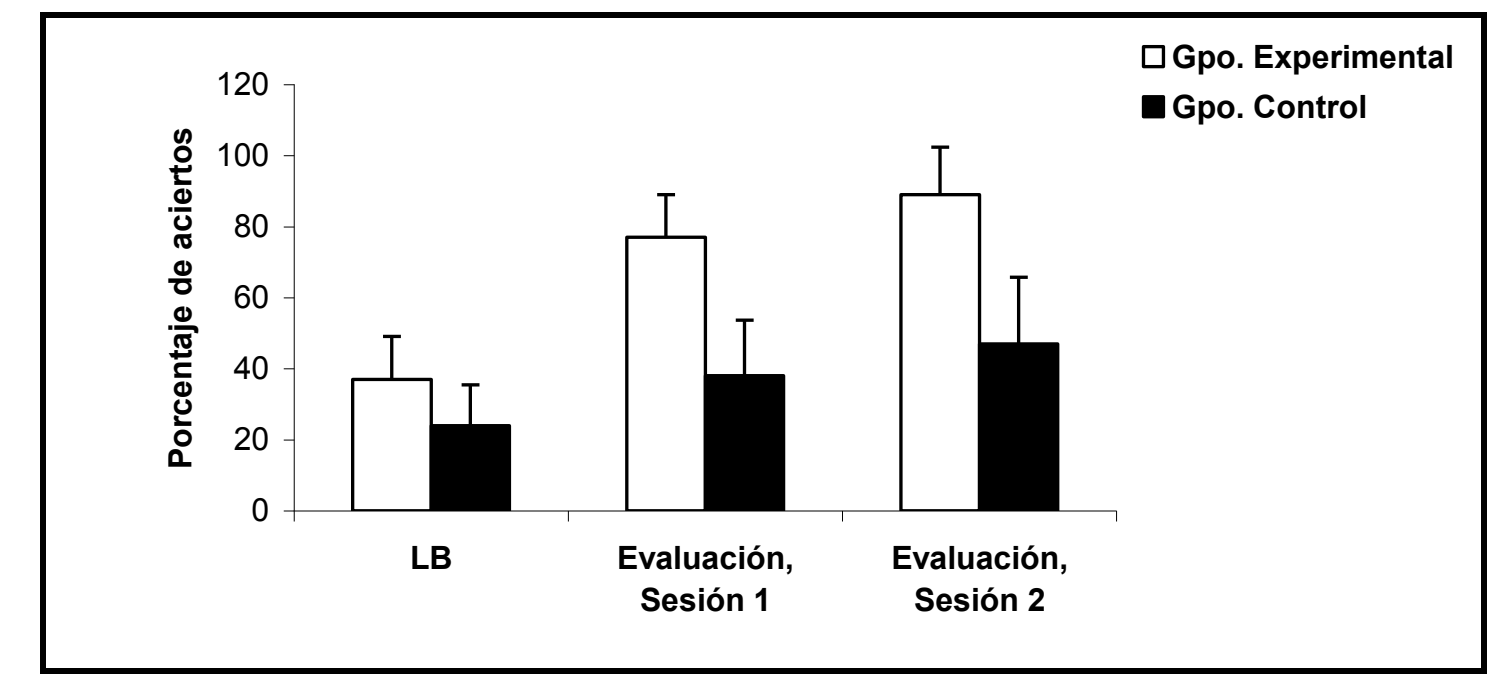

Figura 5. Porcentaje promedio de aciertos obtenido por los participantes de los grupos experimental y control en la fundamentación de la pregunta de investigación que elaboraron en la LB y en las dos sesiones de la Evaluación. Las barras de error representan la desviación estándar.

\section{Discusión}

El objetivo de la presente investigación fue determinar si el entrenamiento en lectura comprensiva y en la composición escrita tenía efectos sobre la elaboración y fundamentación de preguntas de investigación. Específicamente, se esperaba probar si el leer en un nivel extrasituacional promovía o facilitaba el ejercicio de la escritura en dicho nivel.

En primer término, se analizó en qué nivel los participantes elaboraban una pregunta de investigación y cómo la fundamentaban, antes de exponerse a entrenamiento. Se encontró que sólo dos participantes del grupo experimental y ninguno del control fueron capaces de plantear una pregunta de investigación. Y al analizar la fundamentación que hicieron de la pregunta planteada, resaltó el hecho de que todos lo hicieron de manera deficiente.

Respecto a la identificación del tipo de párrafos que componen un artículo científico, llevada a cabo en el Ejercicio 1, se encontró que la mayoría de los participantes tuvieron dificultades para hacerlo, a pesar de que implicaba el nivel más simple de desempeño (situacional). Mientras que en el Ejercicio 2, en el que los participantes debían identificar los tipos de preguntas, identificar variables experimentales y elaborar paráfrasis de lo leído previamente (nivel extrasituacional) se observó que éstos en general tuvieron un desempeño pobre, peor 
aún que el mostrado en el Ejercicio 1. Ello concuerda con lo señalado por Ribes y López (1985) quienes afirman que los niveles implican complejidades crecientes.

Finalmente, en la Evaluación, en la que los participantes debían plantear una pregunta de investigación y su fundamentación, se encontró que en la sesión 1 cuatro participantes del grupo experimental lograron plantear una pregunta de investigación en un nivel de complejidad superior al del nivel de las planteadas por los del grupo control. Ello concuerda con lo señalado por Pacheco et al. (2007), quienes mencionan que para comportarse en los niveles más complejos es requisito contar con un entrenamiento en los niveles inferiores.

Dicho requisito se evidencia en el presente estudio ya que los participantes del grupo experimental, quienes fueron los que se expusieron al entrenamiento, lograron elaborar su pregunta de investigación en niveles que requerían comportarse extrasituacionalmente. Además de que el número de participantes que lograron plantear una pregunta de investigación fue mayor en el grupo experimental que en el control.

Con respecto a la fundamentación de la pregunta de investigación, como puede verse en las figuras 2 y 5 , el grupo experimental tuvo un desempeño signficativamente mejor que el del grupo control, lo que parece indicar que el entrenamiento al que los participantes del grupo experimental fueron expuestos propició una mejora en su ejecución. Respecto a los cambios de la sesión 1 a la 2, se observó un mejor desempeño en la sesión 2 en todos los participantes del grupo experimental, a excepción de un participante que permaneció igual (P1). Ello pudo deberse, como ya se había mencionado, a que a los participantes del grupo experimental se les señalaron puntualmente sus errores al final de la sesión 1, lo que pudo haber propiciado que éstos mejoraran su desempeño en la sesión 2. Sin embargo, también se observó en este aspecto una ganancia en tres participantes del grupo control. Lo anterior pudo deberse a que éstos, al releer su escrito, probablemente identificaron sus errores, lo que les permitió corregirlos.

En general los participantes de ambos grupos mejoraron su ejecución de la LB a la Evaluación, tanto en la elaboración de la pregunta de investigación como en la fundamentación de ésta. Sin embargo, como se muestra en las figuras 2 y 5, se observó una mayor ganancia en ambos aspectos en los participantes del grupo experimental. Podría suponerse que ello se debió al entrenamiento, el cual pudo haber propiciado que los participantes pudieran escri- 
bir en un nivel extrasituacional. Ello concordaría con lo encontrado por Pacheco et al. (2007), de que el nivel en que se ejercita el comportamiento lector corresponde con el nivel en que se ejercita el comportamiento escritor. En este caso se podría decir que probablemente el ejercicio del comportamiento lector en el nivel extrasituacional podría haber promovido que el comportamiento escritor se ejercitara en ese mismo nivel.

Respecto al desempeño de los participantes del grupo control, su mejoría de la LB a la Evaluación podría deberse a que en la primera sólo debieron leer dos artículos para de ahí derivar una pregunta de investigación y fundamentarla, mientras que para el momento de la Evaluación ya habían leído seis artículos mas (los tres empleados en el Ejercicio 1 y los tres del Ejercicio 2), todos ellos relacionados con una misma temática, para, a partir de éstos, derivar una pregunta de investigación debidamente fundamentada.

Lo anterior sugiere que además de los elementos específicamente manipulados en el presente estudio, habría otros que también podrían haber afectado el desempeño de los sujetos, como el relativo a la historia de referencialidad, es decir, qué tan familiar es para el sujeto la temática respecto de la cual debe leer y escribir. Dados los resultados obtenidos, podría ser que la historia de referencialidad de cada sujeto haya afectado la forma y el nivel en el que cada uno de ellos realizó las tareas a las que se les expuso en la presente investigación. Tal variable deberá controlarse en posteriores estudios.

Por otra parte, es necesario considerar la experiencia diferencial con que contaban los participantes en las actividades de investigación, ya que se detectó que algunos habían elaborado tesis de licenciatura y otros habían fungido como auxiliares de investigación por algunos meses (los únicos que no contaban con experiencia fueron los participantes 1 y 3 ). Si bien es cierto que hubiera sido deseable controlar la variable experiencia en investigación, dado que se quería probar el efecto del entrenamiento en todos los alumnos que ingresaron en el mismo ciclo escolar, y a que el número de alumnos que ingresan al posgrado elegido es reducido, dicha variable no se controló.

Otro elemento a considerar es el tipo de texto que se lee. Fuentes (2005) menciona que leer textos científicos implica que el estudiante despliegue comportamientos en distintos niveles, es decir, dependiendo del tipo de texto con el que debe interactuar, el participante deberá desplegar comportamientos en distinto nivel. Tamayo (comunicación personal, 2007) realizó un análisis de los textos utilizados en el presente estudio para determinar el nivel de compleji- 
dad de cada uno y encontró que los textos en los que los participantes tuvieron un peor desempeño eran más complejos (materiales empleados en las sesiones número 2, de los ejercicios 1 y 2) que aquellos en los que tuvieron un mejor desempeño. La complejidad del texto se determinó identificando la claridad con que se exponía en cada artículo la pregunta de investigación y su correspondiente fundamentación. Esta variable también deberá ser contrlada en posteriores estudios.

Otro de los aspectos que pudo haber influido en los resultados obtenidos fue la motivación hacia la tarea por parte de los participantes. Se ha encontrado que los estudiantes motivados tienen un mejor desempeño en las actividades escolares (Pintor y González, 2005). Se observó que dos de los participantes que tuvieron bajo desempeño, uno del grupo control (P11) y otro del grupo experimental (P6), mostraron poca disposición para realizar las tareas. A este respecto vale aclarar que hubiera sido posible influir en la variable motivación hacia la tarea, pero dado que en sólo dos de los participantes se observó este problema, con el fin de mantener la comparabilidad de los datos, se decidió no hacer nada al respecto con el objeto de que todos los sujetos se expusieran exactamente a las mismas condiciones.

Por otra parte, se pudo constatar que, tal y como se suponía, antes del entrenamiento los estudiantes mostraron severas dificultades en la elaboración y fundamentación de preguntas de investigación (Pacheco et al., 2007) y ejercitaron los comportamientos lector y escritor en el nivel mas simple (situacional), y que luego de exponerse al entrenamiento su desempeño mejoró de manera evidente, al lograr comportarse en niveles extrasituacionales. Aunque es necesario destacar el hecho de que en ninguna de las tres sesiones de entrenamiento programadas, ni uno solo de los participantes logró obtener el 100\% de aciertos, a pesar de la retroalimentación recibida en el entrenamiento, pero aún así su desempeño, en lo relativo al nivel en el que plantearon sus preguntas de investigación y las fudamentaron, mejoró considerablemente de la LB a la Evaluación. Dado dicho resultado, se está preparando una réplica no sistemática en la que se expondrá a los participantes a un entrenamiento correctivo, y no solamente a retroalimentación al final de la ejecución, como se hizo en el presente estudio.

Este estudio se planeó así con el objeto de verificar si se confirmaba el supuesto de que la sola retroalimentación mejora la ejecución, tal y como lo señalan diversos autores (Co11, Rochera, Mayordomo y Naranjo, 2007; Ribes y Martínez, 1990). Y el desempeño superior de los participantes del grupo experimental (ver Figura 5), a quienes se les indicaron sus aciertos y errores, pareció confirmar dicho señalamiento. 
Además, los resultados de este estudio concuerdan con lo encontrado previamente por Pacheco et al. (2007), ya que se pudo constatar que el nivel en que se ejercita el comportamiento lector propicia el ejercicio del comportamiento escritor en éste mismo, lo que apunta a la necesidad de proveer entrenamiento lector en niveles complejos a los científicos en formación. El que los participantes fueran capaces de plantear una pregunta de investigación debidamente fundamentada en la Evaluación parece indicar que éstos fueron capaces de ejercitar la escritura en un nivel extrasituacional después de exponerse al entrenamiento.

Aparentemente el exponer a investigadores en formación a la lectura de materiales técnicos, escritos con un lenguaje complejo, y entrenarlos de manera explícita para escribir textos científicos parece promover que éstos mejoren su desempeño al elaborar y justificar preguntas de investigación derivadas de artículos previamente leídos. Tales datos parecen apuntar a la pertinencia de proveer entrenamiento lector y escritor a los científicos en formación empleando materiales complejos.

Finalmente, es importante señalar que una limitación de este estudio podría ser el pequeño número de participantes, sin embargo, no se pudo trabajar con más debido a que el posgrado al que pertenecían, dada su orientación tutorial, sólo acepta un grupo pequeño de alumnos por ciclo escolar. Por eso se planea llevar a cabo más estudios, conforme ingresen otros grupos. Aunque los resultados obtenidos en el presente trabajo parecen indicar que es factible, empleando el procedimiento aquí propuesto, mejorar el desempeño escritor de los participantes, habilidad crítica para todo investigador.

Por otra parte, con el objeto de seguir analizando las variables que afectan el planteamiento de preguntas de investigación, quedaría pendiente llevar a cabo estudios en los que se exponga a los participantes a un entrenamiento con distintas tareas que propicien la elaboración de preguntas de investigación en diferentes niveles de complejidad. Así como diseñar preparaciones experimentales en las que se evalúe el efecto de distintos aspectos como el tipo de texto empleado, la complejidad de éste, y lo relativo a la historia referencial del sujeto, entre otros. 


\section{Referencias}

Canales, C., Morales, G., Arroyo, R., Pichardo, A. y Pacheco, V. (2005). Análisis Interconductual del ajuste lector en el ámbito educativo. En C. Carpio y J.J. Irigoyen (Eds.) Psicología y educación. Aportaciones desde la teoría de la conducta (pp. 33-50). México: UNAM.

Carpio, C. (1994). Comportamiento animal y teoría de la conducta. En: L. Hayes, E. Ribes y F. López (Coords.), Psicología interconductual: Contribuciones en honor a J.R. Kantor (pp. 45-68). México: Universidad de Guadalajara.

Carpio, C. y Irigoyen, J.J. (2005). Psicología y educación. Aportaciones desde la teoría de la conducta. México: UNAM.

Carpio, C., Pacheco, V., Flores, C. y Canales, C. (2000). La naturaleza conductual de la comprensión. Revista Sonorense de Psicología, 14, 1, 25- 34.

Cassany, D. (2006). Taller de textos. Leer, escribir y comentar en el aula. España: Paidós.

Cassany, D. (2007). Evaluación de textos escritos. Curso impartido en el Instituto Tecnológico de Monterrey, Monterrey, Nuevo León, México.

Coll, S.C., Rochera, V.M.J., Mayordomo S.R.M. y Naranjo, L1.M. (2007). Evaluación continua y ayuda al aprendizaje. Análisis de una experiencia de innovación en educación superior con apoyo de las TIC. Electronic Journal of Research in Educational Psychology, 13, 5(3), 783-804.

De la Fuente, J., Justicia, F., Casanova, P.F., y Trianes, M.V. (2005). Perceptions about the construction of academic and professional competencies in psychologist. Electronic Journal of Research in Educational Psychology, 3(1), 3-34.

Fuentes, T. (2005). Repertorios recurrentes de la comprensión lectora reconstructiva. Tesis doctoral inédita. Universidad de Guadalajara.

Keys, C.W., Hand, B., Prain, V., y Collins, S. (1999). Using the science writing heuristic as a tool for learning from laboratory investigations in secondary science. Journal of Research in Science Teaching, 36(10), 1065-1084.

Irigoyen, J.J., Jiménez, M. y Acuña, K. (2004). Evaluación del ejercicio instruccional en la enseñanza universitaria. Enseñanza e Investigación en Psicología, 9(2), 293-302.

Pacheco, V. (2008). Generación de conocimiento y habilidades escritoras: un análisis de la producción de textos en universitarios. Tesis doctoral inédita. Universidad Nacional Autónoma de México.

Pacheco, V., Ramírez, L., Palestina, L., y Salazar, M. (2007). Una aproximación al análisis funcional de la relación entre las conductas de leer y escribir en estudiantes de psico- 
logía. En J.J. Irigoyen, M. Jiménez y K. Acuña (Eds.), Enseñanza, aprendizaje y evaluación. Una aproximación a la pedagogía de las ciencias (pp. 247-275). México: UniSon.

Pintor, M. y González, P. (2005). La motivación en secundaria, un estudio empírico. Revista Complutense de Educación, 16, 339-352.

Ribes, E. (1990). Psicología General. México: Trillas.

Ribes, E. (2004). Acerca de las funciones psicológicas. Un post-scriptum. Acta Comportamentalia, 12(2), 117-128.

Ribes, E., Cortés, A. y Romero, P. (1992). Quizá el lenguaje no es un proceso o tipo especial de comportamiento: algunas reflexiones basadas en Wittgenstein. Revista Latina de Pensamiento y Lenguaje, 1(1), 58-74.

Ribes, E. y López, F. (1985). Teoría de la conducta, un análisis de campo y paramétrico. México: Trillas.

Ribes, E. y Martínez, H. (1990). Interaction of contingencies and rule instructions in the performance of human subjects in conditional discrimination. The Psychological Record, $40,565-586$.

Sánchez, P.R. (2004). Enseñar a investigar. Una didáctica nueva de la investigación en ciencias sociales y humanas. México: Plaza y Valdés.

Viniegra, L. (2002). Un acercamiento a la crítica. Educación y Crítica: el proceso de elaboración del conocimiento. México: Paidós. 


\section{Anexo 1. Listado de los artículos a los que se expuso a los participantes en la presente investigación.}

Carpio, C., Pacheco, V., Carranza, N., Flores, C. \& Canales, C. (2003). Tipos de retroalimentación en el aprendizaje de términos metodológicos de la psicología experimental. Anales de Psicología, 19, 1, 97-105.

Carpio, C., Silva, H., Lada, E., Morales, G., Arroyo, R., Canales, C. \& Pacheco, V. (2006). Generación de criterios de igualación: Un caso de conducta creativa. Universitas Psychologica, 5, 1, 127-138.

Ferro, R., \& Valero, L. (2005). Formación de categorías pictóricas a través de relaciones de equivalencia. Psicothema, 17, 1, 83-89.

Irigoyen, J., Carpio, C., Jiménez, M., Silva, H., Acuña, K. \& Arroyo, A. (2002). Variabilidad en el entrenamiento en retroalimentación parcial en la adquisición de desempeños efectivos y su transferencia. Enseñanza e Investigación en Psicología, 7, 2, 221-234.

Luciano, M.C. \& Valero, L. (1997). Discriminación condicional en niños: Los efectos de contingencias diferenciales de reforzamiento/tiempo fuera. Psicothema, 9, 3, 509-608.

Ribes, E., Torres, C. \& Barrera, J. (1995). Interacción del tipo de entrenamiento, morfología de la respuesta y demora de la retroalimentación en la adquisición y transferencia de la ejecución en una tarea de igualación a la muestra de primer orden en humanos. Revista Mexicana de Análisis de la Conducta, 21, 145-164. 